\title{
Optical spectroscopy of trivalent chromium in sol-gel lithium niobate
}

\author{
J. K. Krebs \\ Dept. of Physics and Astronomy, Franklin and Marshall College, Lancaster, PA 17604 \\ U. Happek \\ Dept. of Physics and Astronomy, The University of Georgia, Athens, GA 30602-2451
}

(Dated: October 9, 2018)

\begin{abstract}
We report on the characterization of sol-gel derived lithium niobate via trivalent chromium probe ions, a study that is motivated by recent reports on the synthesis of high quality sol-gel lithium niobate $\left(\mathrm{LiNbO}_{3}\right)$. In order to assess the quality of sol-gel derived $\mathrm{LiNbO}_{3}$, we incorporate $\mathrm{Cr}^{3+}$ during the hydrolysis stage of the sol-gel process. A comparison of the $\mathrm{Cr}^{3+}$ emission and photoexcitation data on both sol-gel and melt-grown $\mathrm{LiNbO}_{3}$ shows that the sol-gel derived material is highly stoichiometric.
\end{abstract}

PACS numbers: $78.55 \mathrm{Hx}, 71.55 \mathrm{Ht}, 71.70 \mathrm{Ch}, 77.84 \mathrm{Bw}$

The need for sol-gel derived ferroelectric coatings and films for electro-optical and piezo-electric applications is driving the research for high quality sol-gel lithium niobate $\left(\mathrm{LiNbO}_{3}\right)$ 1, 2] . Recent reports concentrate on optimizing the sol-gel process with the aim of producing high quality films with preferred orientation. X-ray diffraction and ICP have been the primary techniques to assess stoichiometry of the samples. To our knowledge, optical probes, such as $\mathrm{Cr}^{3+}$ ions, have not been used to characterize the sol-gel samples, although this approach has been very successful to show fundamental differences between congruent and stoichiometric melt-grown $\mathrm{LiNbO}_{3}$ [3]. The $\mathrm{Cr}^{3+}$ ion is particulary suited to study the quality of $\mathrm{LiNbO}_{3}$, because it is arguably the most studied optical impurity [4], has a spectrum that is very sensitive to the possible lattice sites and disorder in $\mathrm{LiNbO}_{3}$, and can be readily incorporated in an early stage of the sol-gel process. In addition, chromium doped lithium niobate has stimulated much interest due to the broadband near infrared luminescence of the chromium ions, with the ultimate goal of developing a tuneable laser in the visible range through frequency doubling within the active medium.

In the current work, we study the optical $\mathrm{Cr}^{3+}$ transitions between the ${ }^{4} A_{2}$ groundstate and the lowest ${ }^{4} T_{2}$ and ${ }^{2} E$ excited electronic states. These transitions are characterized by broad absorption and emission bands for the spin allowed transition, and spectrally narrow lines for the spin-forbidden transition, respectively. These transitions provide information on the stoichiometry and disorder of the sol-gel material, and allow a comparison to the detailed study of $\mathrm{LiNbO}_{3}$ samples grown from melt.

For the sample synthesis, we combine $1 \mathrm{M}$ solutions of lithium and niobium ethoxides in ethanol such that the ratio of $\mathrm{Li}: \mathrm{Nb}$ is 1:1. An appropriate amount of $\mathrm{Cr}\left(\mathrm{NO}_{3}\right)_{3}$ is then dissolved in water (in this study the $\mathrm{Cr}: \mathrm{Li}$ ratio was 0.001:1). Under constant stirring, the water-chromium solution is slowly added into the mixed ethoxide. After an initial release of ethanol, the solution is capped and aged for several days. The aged solution is then dried at room temperature until a fine white powder

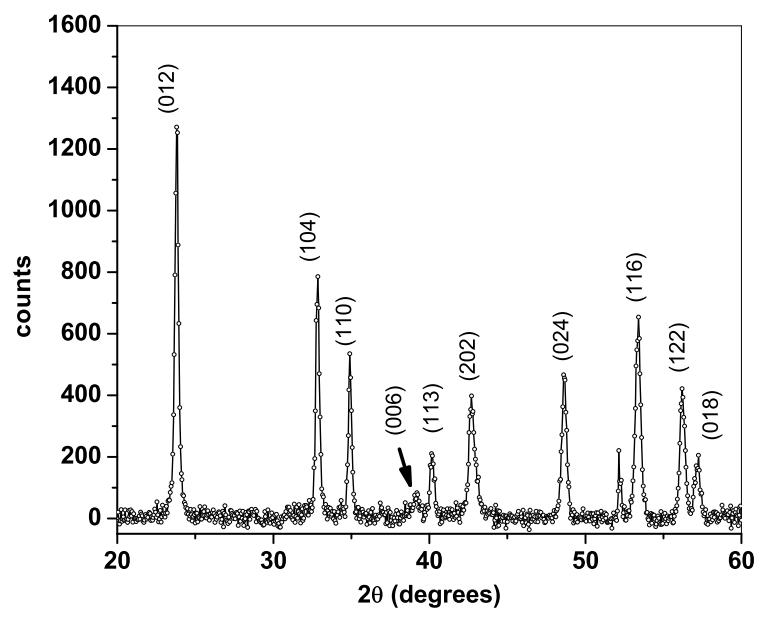

FIG. 1: X-ray diffraction pattern of sol-gel $\mathrm{LiNbO}_{3}: 0.1 \% \mathrm{Cr}^{3+}$.

has formed. The powder samples are finally heated in air to $800{ }^{\circ} \mathrm{C}$ for two hours to remove excess water and organic complexes. The calcined powders are characterized by $\mathrm{x}$-ray diffraction utilizing $\mathrm{Cu} \mathrm{K}$-alpha radiation; a typical pattern is shown in Fig. 1. All observed diffraction peaks can be attributed to lithium niobate, and we find no evidence for alternate phases such as $\mathrm{Li}_{0.88} \mathrm{H}_{0.12} \mathrm{NbO}_{3}$.

The optical experiments are carried out with the powder sample mounted in an Oxford Instruments temperature variable cryostat. For emission measurements the sample is excited with a HeNe laser at $632.8 \mathrm{~nm}$. The broadband emission was collected with reflective optics and analyzed with a Bruker 66v FTIR spectrometer. Photoexcitation measurements were performed by mounting the cryostat directly into the sample space of a modified Cary 14 spectrophotometer. In both the broadband emission and photoexcitation experiments, the emitted light was collected through appropriate filters and imaged onto a liquid-nitrogen cooled InGaAs detector. For the photoexcitation, a lock-in amplifier was used 


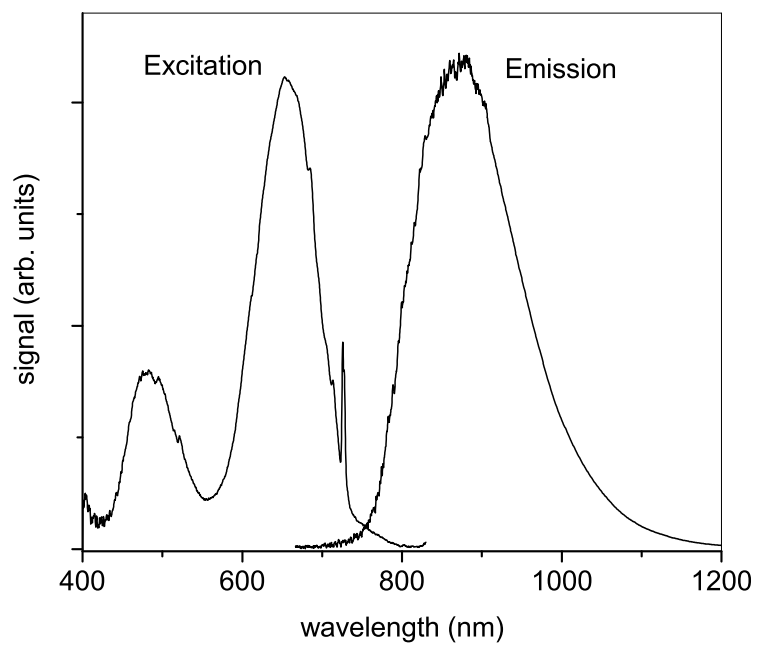

FIG. 2: Excitation and emission spectra from sol-gel $\mathrm{LiNbO}_{3}$ : $0.1 \% \mathrm{Cr}^{3+}$.

for signal-to-noise enhancement.

Experimental results for the sol-gel $\mathrm{LiNbO}_{3}: 0.1 \% \mathrm{Cr}^{3+}$ sample are shown in Fig. 2. The excitation spectrum (Fig.2, left) observed at $\lambda_{\text {det }} \geq 900 \mathrm{~nm}$ shows the characteristic ${ }^{4} \mathrm{~A}_{2} \rightarrow{ }^{4} \mathrm{~T}_{2}$ and ${ }^{4} \mathrm{~A}_{2} \rightarrow{ }^{4} \mathrm{~T}_{1}$ broad absorption bands around $655 \mathrm{~nm}$ and $480 \mathrm{~nm}$, respectively, as well as the spin-forbidden ${ }^{4} \mathrm{~A}_{2} \rightarrow{ }^{2} \mathrm{E}$ transition around 726 $\mathrm{nm}$. The emission spectrum (Fig. 2, right) shows the ${ }^{4} \mathrm{~T}_{2} \rightarrow{ }^{4} \mathrm{~A}_{2}$ emission band under HeNe laser excitation $\left(\lambda_{e x}=632.8 \mathrm{~nm}\right)$.

To interpret these results, they are compared to spectra obtained on standard melt-grown $\mathrm{LiNbO}_{3}: \mathrm{Cr}^{3+}$. Lithium niobate crystals pulled from a melt with equal amounts of lithium and niobium crystallize with a $\mathrm{Li}$ $3 \%$ deficit [5], and are referred to as congruent samples. When chromium ions are added to these melts, the trivalent impurities preferentially occupy the crystallographic site of lithium vacancies, in the following referred to as $\mathrm{Cr}[\mathrm{Li}]$. The crystal field leads to strong absorption into the ${ }^{4} \mathrm{~T}_{2}$ and ${ }^{4} \mathrm{~T}_{1}$ levels peaking at 654 $\mathrm{nm}$ and $478 \mathrm{~nm}$, respectively, giving the crystals a green color. A shift in the optical spectra is observed when $\mathrm{LiNbO}_{3}: \mathrm{Cr}^{3+}$ is codoped with more that $4.5 \% \mathrm{Mg}^{2+}$, resulting in $\mathrm{Cr}^{3+}$ impurities preferentially occupying niobium sites [6]. The resulting change in the crystal field shifts the ${ }^{4} \mathrm{~T}_{2}$ and ${ }^{4} \mathrm{~T}_{1}$ absorption peaks to $715 \mathrm{~nm}$ and $540 \mathrm{~nm}$, resulting in a crystal with a red color. New crystal growth techniques, namely high-temperature topseeded solution growth methods (HTTSSG), enabled the production of $\mathrm{LiNbO}_{3}$ crystals that are stochiometric (i.e. no $\mathrm{Li}$ or $\mathrm{Nb}$ deficit) []]. These samples produce spectra with narrower linewidths enabling more precise optical spectroscopy and better characterization of the various chromium sites [3], moreover, the chromium ions in these stoichiometric melt-grown single crystals are found to oc-

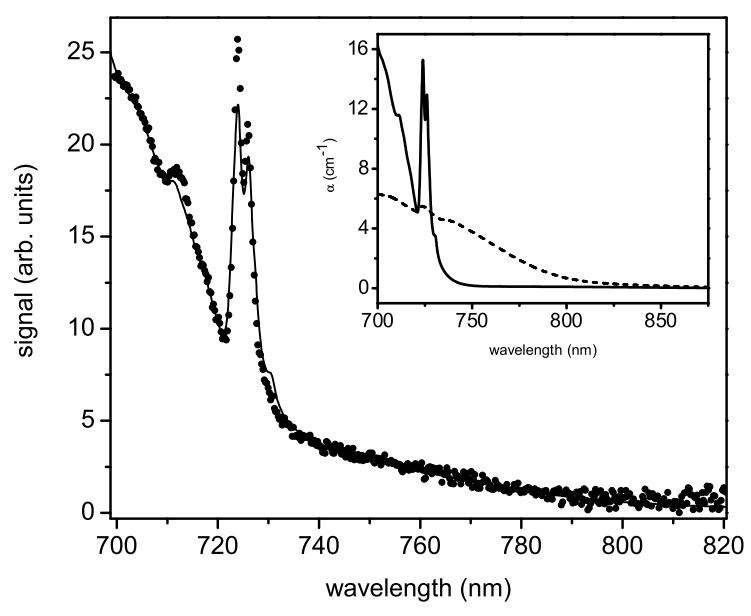

FIG. 3: Excitation of sol-gel $\mathrm{LiNbO}_{3}: 0.1 \% \mathrm{Cr}^{3+}$ (circles). The inset shows the absorption coefficient of $\mathrm{Cr}^{3+}$ ions in single crystal samples of $\mathrm{LiNbO}_{3}$ and $\mathrm{LiNbO}_{3}: 6 \% \mathrm{Mg}$. The fit to the excitation spectrum determines the $66 \% \mathrm{Cr}[\mathrm{Li}]$ to $34 \% \mathrm{Cr}[\mathrm{Nb}]$ ratio.

cupy both Li and $\mathrm{Nb}$ sites [8].

In contrast to the $\mathrm{Cr}[\mathrm{Li}]$ spectrum that dominates in congruent $\mathrm{LiNbO}_{3}$ crystals, the sol-gel produced material has a significant contribution to the luminescence at excitation wavelengths beyond $750 \mathrm{~nm}$, as evident in a shoulder in the excitation spectrum extending from about $750 \mathrm{~nm}$ to $800 \mathrm{~nm}$ in Fig.2. Fig.3 shows an expanded excitation spectrum in this region (dots). This shoulder can be attributed to chromium ions on niobium sites, $\mathrm{Cr}[\mathrm{Nb}]$. To quantify this contribution to the excitation spectrum, we measured the spectra of $\mathrm{LiNbO}_{3}: \mathrm{Cr}^{3+}$ crystals with and without magnesium codoping (6\%).

The inset of Fig. 3 shows the absorption for the single crystal samples of $\mathrm{LiNbO}_{3}: \mathrm{Cr}$ (solid) and $\mathrm{LiNbO}_{3}: \mathrm{Cr}: \mathrm{Mg}$ (dashed) in the $700 \mathrm{~nm}$ to $850 \mathrm{~nm}$ range, clearly demonstrating the difference of the ${ }^{4} \mathrm{~T}_{2}$ onset for the two sites. The fit to the sol-gel data (Fig. 3, main plot, line) was found by varying the contributions to the impurity absorption for each site while keeping the total constant, i.e $\alpha_{\text {sol-gel }}=\mathrm{C}_{L i} \alpha_{C r[L i]}+\mathrm{C}_{N b} \alpha_{C r[N b]}$, with $\mathrm{C}_{L i}+\mathrm{C}_{N b}$ $=1$. The fit shown in Fig.3 (solid line) represents a $\mathrm{C}_{L i}=0.66$ contribution from the $\mathrm{Cr}[\mathrm{Li}]$ and a $\mathrm{C}_{N b}=$ 0.34 contribution from $\mathrm{Cr}[\mathrm{Nb}]$. This approach is justified because our single crystal reference samples contained the same amount of chromium ions $(0.25 \%)$. A careful inspection of $\mathrm{LiNbO}_{3}: \mathrm{Cr}: \mathrm{Mg}$ (insert Fig.3, dashed line) shows a small peak at $727 \mathrm{~nm}\left({ }^{4} \mathrm{~A}_{2} \rightarrow{ }^{2} \mathrm{E}\right.$ transition), due to $\mathrm{Cr}[\mathrm{Li}]$. Taking this correction into account, we arrive at relative $\mathrm{Cr}[\mathrm{Li}]$ and $\mathrm{Cr}[\mathrm{Nb}]$ concentrations of 0.64 and 0.36 , respectively.

The substantial occupation of the niobium site by chromium ions is in marked difference to melt-grown congruent samples, where the chromium ions occupy lithium 
sites exclusively. In highly stoichiometric $\mathrm{LiNbO}_{3}$ samples, grown by the HTTSSG method [7], we also find chromium ions on both the lithium and niobium sites [8], thus our results confirm reports that the sol-gel process yields highly stoichiometric samples without the intrinsic Li deficit, which is characteristic for congruent meltgrown $\mathrm{LiNbO}_{3}$. In stoichiometric single crystal samples of highest quality, the zero-phonon line of the ${ }^{4} \mathrm{~A}_{2} \rightarrow{ }^{4} \mathrm{~T}_{2}$ can be observed at low temperature. In our sample, this transition is broadened, which is indicative of lattice defects. Our sample also shows the optical signature of so-called high field sites, which is due to $\mathrm{Cr}^{3+}$ ions in a strong crystal field, resulting in the ${ }^{2} \mathrm{E}$ level lying below the ${ }^{4} \mathrm{~T}_{2}$ level, characterized by sharp emission lines at low temperatures, and which are associated with defects in the crystal. Thus, while our results indicate that the sol-gel samples do not have the intrinsic lithium deficit of congruent melt-grown material, the overall quality of the material does not yet approach that of the highest quality single crystal material. However, with the optical absorption and emission spectra of trivalent chromium being a clear indicator of the crystal quality, systematic studies are possible to improve the quality of sol-gel derived $\mathrm{LiNbO}_{3}$.

In summary, we have shown $\mathrm{Cr}^{3+}$ ions are sensitive optical probes of the quality of sol-gel derived $\mathrm{LiNbO}_{3}$. Our experiments confirm that sol-gel produced lithium niobate is highly stoichiometric and does not contain the intrinsic $\mathrm{Li}$ deficit of congruent $\mathrm{LiNbO}_{3}$. Our results also show that our sol-gel material contains more defects than high quality stoichiometric $\mathrm{LiNbO}_{3}$. With the aid of the chromium optical probe ions, experiments are under way to improve the quality of sol-gel $\mathrm{LiNbO}_{3}$ and related ferroelectric materials.
[1] Shin-ichi Hirano, Y. Takeichi, W. Sakamoto, and T. Yogo, Journal of Crystal Growth 237-239, 2091 (2002).

[2] S. Cheng, X. Han, C. Kam, Y. Zhou, Y. Lam, J. T. Oh, X. Xu, and T. Chong, Applied Physics A 73, 511 (2001).

[3] G. Salley, S. Basun, A. Kaplyanskii, R. Meltzer, K. Polgar, and U. Happek, Journal of Luminescence 87-89, 1133 (2000).

[4] B. Henderson and G. Imbusch, Optical Spectroscopy of Inorganic Solids (Oxford University Press, Clarendon, Oxford, 1989).
[5] R. Byer, J. Young, and R. Feigelson, Journal of Applied Physics 41, 2320 (1970).

[6] P. I. Macfarlane, K. Holliday, J. F. Nicholls, and B. Henderson, Journal of Physics: Condensed Matter 7, 9643 (1995).

[7] K. Polgar, A. Peter, L. Kovacs, G. Corradi, and Z. Szaller, Journal of Crystal Growth 177, 211 (1997).

[8] G. Salley, Ph.D. thesis, The University of Georgia (2000). 